\title{
ВЛИЯНИЕ УПСГ НА ЭФФЕКТИВНОСТЬ ЭНЕРГЕТИЧЕСКИХ УСТАНОВОК СУПЕРГАЗОВОЗОВ
}

\author{
Голубев P.O. Email: Golubev1141@scientifictext.ru
}

\author{
Голубев Роман Олегович - бакалавр, студент, \\ кафедра судовых энергетических установок, систем и оборудования, \\ Санкт-Петербургский государственный морской технический университет, г. Санкт-Петербург
}

\begin{abstract}
Аннотация: в статье определяется ранее не рассматривавшаяся проблема влияния бортовой УПСГ на эффективность газовозов рефрижераторного типа. Устанавливается причина значительного влияния этого фактора на эффективность эксплуатаиии судов типа. Предлагается критерий и условия оченки влияния УПСГ на эффективность СЭУ. Формулируются математические модели совместного влияния ГД и УПСГ на экономичность 4-х типов СЭУ, на базе супергазовоза вместимостью 210 тыс. м $^{3}$ СПГ. На основании предложенного критерия проводится сравнительный анализ этих СЭУ.

Ключевые слова: вспомогательная энергетическая установка (ВЭУ), главный двигатель (ГД), главный котёл (ГК), главная энергетическая установка (ГЭУ), дизельная энергетическая установка (ДЭУ), малооборотный дизель (МОД), природный газ (ПГ), промежуточный перегрев пара (ППП), паротурбогенератор (ПТГ), паротурбинная установка (ПТУ), сжиженный природный газ (СПГ), судовая энергетическая установка (СЭУ), тяжёлое топливо (ТТ), установка повторного сжижения газа (УПСГ).
\end{abstract}

\section{INFLUENCE OF ONBOARD RELIQUEFACTION PLANT UPON EFFICIENCY OF SUPER LNG CARRIER'S POWER PLANT Golubev R.O.}

\author{
Golubev Roman Olegovich - Bachelor of Science, Student, \\ DEPARTMENT OF MARINE POWER PLANTS SYSTEMS AND EQUIPMENT, \\ SAINT-PETERSBURG STATE MARINE TECHNICAL UNIVERSITY,
} SAINT-PETERSBURG

\begin{abstract}
LNG carrier's efficiency dependence of onboard reliquefaction plant. Cause of this problem`s influence upon ship`s maintenance efficiency was described. Crucial criterion and estimation conditions of reliquefaction plant's influence upon ship power plant's efficiency are represented. Mathematical models of main engine and reliquefaction plant coworking were created for four types of super 210000 cubic meter LNG carrier`s power plants. Comparative study of plants was carried on. Survey was based on criterion offered.

Keywords: auxiliary power plant, boiled off gas reliquefaction plant, diesel power plant, heavy fuel oil, liquefied natural gas, low speed diesel engine, main boiler, main engine, main power plant, marine power plant, natural gas, reheat steam facility, steam-driven generator set, steam turbine power plant.
\end{abstract}

УДК 621.592 .3

\section{Причина влияния УПСГ на пропульсивную установку газовоза СПГ}

Под супергазовозами в статье понимаются суда грузовместимостью на уровне газовозов семейства Qflex и более. На таких судах часто возникает проблема избыточности выпара груза. Одним из эффективных путей её решения служит УПСГ. Использование УПСГ в составе СЭУ газовозов СПГ - это относительно новая тенденция в развитии энергетики судов этого типа. Активное внедрение УПСГ началось в 2007 году со спуском на воду первых газовозов семейства Q-flex [3, с. 4]. Такие установки, работающие на азоте (хладагенте) по обратному (холодильному) циклу Брайтона, потребляют порядка 28 кВт электрической мощности на тысячу $\mathrm{m}^{3}$ полной грузовместимости. Наличие в составе ВЭУ такого крупного потребителя энергии оправдывается высокими ценами на природный газ и подразумевает работу ГЭУ исключительно на нефтяном топливе. Несмотря на то, что мощность УПСГ, примерно, составляет $20 \%$ от мощности ГД, влияние перераспределения вырабатываемой СЭУ энергии между ГЭУ и ВЭУ на эксплуатационные характеристики газовоза осталось неучтённым - газовозы Q-flex и последующие серии газовозов сохранили эксплуатационную скорость хода на прежнем уровне (в районе 20 узлов).

\section{Методика оценки влияния УПСГ на типы СЭУ газовозов СПГ}

В качестве базового варианта принимается газовоз с полной грузовместимостью $\left(W_{\text {полн }}\right) 210$ тыс. ${ }^{3}$ СПГ, оснащённый ПТУ с ППП эффективной мощностью $\left(N_{e}^{\sigma}\right) 26130$ кВт, удельным эффективным расходом топлива $\left(g_{e}\right) 0,245$ Кг /кВт · ч и имеющий эксплуатационную скорость хода (соответствующую $\left.N_{e}^{\sigma}\right) 20$ уз $\left(v_{б}\right)$, УПСГ на борту отсутствует. 
Рассматривается влияние УПСГ на следующие типы СЭУ: ПТУ с ППП, ДЭУ с МОД МЕ (MAN $\mathrm{B} \& \mathrm{~W}$ ) - и ДЭУ с МОД ME-GI (MAN B\&W) без УПСГ как альтернатива - работающие на ТТ и СПГ. В базовой модели выпар используется в ГК ПТУ, а его избыток реализуется в ВЭУ, например, для выработки электроэнергии ПТГ. При наличии УПСГ, для ПТУ и ДЭУ с МОД МЕ учитываются дополнительные затраты ВЭУ по топливу на её привод. ГД работают на ТТ без добавления выпара, а весь выпар повторно сжижается. ДЭУ с МОД ME-GI - работает на смеси ТТ+ПГ и, по режиму использования ПГ, подобна базовому варианту.

В качестве оценочного критерия принимается среднечасовая стоимость затраты энергоресурса на ГД и УПСГ $\left(C_{\ni}=\$ /\right.$ ч $) . C_{э}$ определяется по формуле 1.

$$
C_{\ni}=S_{\text {уд }}^{\mathrm{TT}} B_{\mathrm{TT}}+S_{\text {уд }}^{\Pi \Gamma} B_{\text {ПГ }}
$$

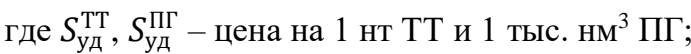

$B_{\mathrm{TT}}, B_{П Г}-$ среднечасовой расход ТТ и ПГ, соответственно, в нт и тыс. нм ${ }^{3}$. В случае избыточности неутилизируемого выпара подставляется его полный часовой объём.

Этот критерий физичен, прост для понимания и проявляет значительную чувствительность к изменению типа СЭУ. Сравнение вариантов проводится при условии неизменности интенсивности грузоперевозки. Условие выражается формулой 2.

$$
v_{6} W_{\text {полез }}^{\text {б }}=v_{i} W_{\text {полез }}^{i}=\text { const }
$$

где $v_{i}$ - эксплуатационная скорость хода і-го варианта;

$W_{\text {полез }}^{\text {б }}, W_{\text {полез }}^{i}-$ полезная грузовместимость базового и і-го вариантов.

Формулирование расчётных моделей

ПТУ с ППП без УПСГ (базовый вариант)

Полезная грузовместимость базового варианта:

$$
W_{\text {полез }}^{\text {б }}=W_{\text {полн }}\left(1-k_{\text {охл }}-\frac{k_{\mathrm{B}}^{\text {гр }} S}{24 v_{\text {б }}}\right)
$$

где $k_{\text {охл }}$ - доля от полного объёма груза, оставляемая в танках для поддержания температурного режима на балластном переходе (при отсутствии УПСГ $-0,05$ );

$k_{\mathrm{B}}^{\text {гр }}$ - доля суточных испарений при ходе в грузу $(0,0015)[2$, с.106];

$S$ - плечо рейса.

Доля СПГ в котельной топливной смеси при ходе в грузу и балласте:

$$
\mathrm{y}_{\text {гр }}=\text { Убал }_{\text {ба }}=0,9
$$

Значение принимается исходя из того, что для супергазовозов получаемое количество выпара будет превышать потребное на пропульсию, а при сжигании ПГ в ГК процент нефтяного топлива должен быть не ниже 10. Доля тяжёлого топлива определяется как: $x=1-y$.

Коэффициент калорийности топливной смеси ГК:

$$
k_{Q}^{\Pi \Gamma+\mathrm{TT}}=\frac{Q_{\mathrm{p}}^{\mathrm{H}}{ }^{\mathrm{LT}}}{x Q_{\mathrm{p}}^{\mathrm{TT}}+y Q_{\mathrm{p}}^{\mathrm{H} \Gamma}}
$$

где $Q_{\mathrm{p}}^{\mathrm{H}}{ }^{\mathrm{C}}, Q_{\mathrm{p}}^{\mathrm{HT}}, Q_{\mathrm{p}}^{\text {нТТ }}$ - массовая удельная низшая теплота сгорания ПГ, ТТ и дизельного топлива (стандартного).

Среднечасовой расход тяжёлого топлива:

$$
B_{\mathrm{TT}}=\frac{x g_{e} N_{e}^{\sigma} k_{Q}^{\Pi \Gamma+\mathrm{TT}}\left(1+k_{\text {бал }}\right)}{2 \cdot 10^{3}}
$$

где $k_{\text {бал }}-$ коэффициент снижение мощности ГД на балластном переходе $(0,788)$.

Среднечасовой расход ПГ:

$$
B_{\text {ПГ }}=\frac{k_{\rho} W_{\text {полн }}}{2 \cdot 10^{3}}\left(\frac{k_{\text {охл }} v_{б}}{S}+\frac{k_{\mathrm{B}}^{\text {гр }}}{24}\right)
$$

$k_{\rho}$ - коэффициент плотности, учитывает уменьшение плотности ПГ при нормальных условиях. ПТУ с ППП с УПСГ (вариант 1)

Полезная грузовместимость:

$$
W_{\text {полез }}=W_{\text {полн }}\left(1-k_{\text {охл }}\right)
$$

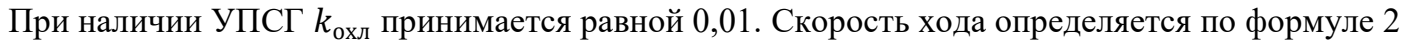
Коэффициент калорийности топлива:

Среднечасовой расход тяжёлого топлива:

$$
k_{Q}^{\mathrm{TT}}=Q_{\mathrm{p}}^{\mathrm{H} Д \mathrm{~T}} /{ }_{Q_{\mathrm{p}}^{\mathrm{H}}}
$$




$$
B_{\mathrm{TT}}=\frac{k_{Q}^{\mathrm{TT}}}{10^{3}}\left[\frac{g_{e} N_{e}^{\sigma}\left(1+k_{\text {бал }}\right) v^{3}}{2 v_{\text {б }}^{3}}+\frac{g_{e}^{\text {ВД }} N_{\text {уд }} k_{\mathrm{B}}^{\text {гр }} W_{\text {полн }} k_{\mathrm{M}}}{24 \eta_{\text {эл }}}\right]
$$

где $g_{e}^{\text {ВД }}-$ удельный расход топлива приводом электрогенератора на генерирование электроэнергии потребной УПСГ (0,3 кГ/кВт · ч для ПТГ);

$N_{\text {уд }}$ - потребная электрическая мощность на $1 \mathrm{~m}^{3} / ч$ СПГ $\left(410 \mathrm{\kappa BT} \cdot \mathrm{q} / \mathrm{M}^{3}\right)$ [1, с.80];

$k_{\mathrm{M}}$ - коэффициент, учитывающий влияние морских условий эксплуатации на грузообработку (волнение, внутрижидкостное трение), возможное увеличение производительности при ходе в балласте (поскольку второе слагаемое принимается осреднённым на переходы в грузу и балласте), $k_{\mathrm{M}}=1,08$;

$\eta_{\text {эл }}-$ коэффициент эффективности электропередачи $(0,95)$.

Среднечасовой расход ПГ:

\section{ДЭУ с МОД МЕ с УПСГ (вариант 2)}

$$
B_{\text {ПГ }}=\frac{k_{\rho} k_{\text {охл }} W_{\text {полн }} v}{2 S \cdot 10^{3}}
$$

Это вариант не имеет принципиальных отличий от предыдущего по схеме грузообработки. Поэтому, формулы 8, 9, 10, 11 для него остаются актуальными. Тем не менее для МОД свойственен меньший $g_{e}$

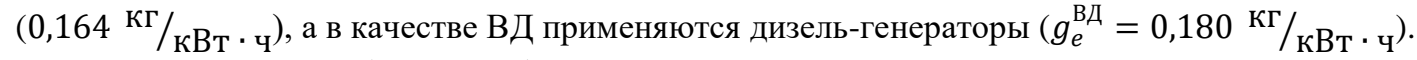

ДЭУ с МОД МЕ-GI (вариант 3)

При отсутствии УПСГ, $k_{\text {охл }}=0,05$, и $W_{\text {полез }}$ определяется по формуле 3 . Следовательно, скорость хода равна базовой. Формула 5 объективна, но при иных значениях коэффициентов $x$ и у, что объясняется организацией процесса сгорания топлива в ДВС $(\mathrm{y}=0,95)$.

Среднечасовой расход тяжёлого топлива:

$$
B_{\mathrm{TT}}=\frac{x g_{e} k_{\text {КВд }} N_{e}^{\sigma} k_{Q}^{\Pi \Gamma+\mathrm{TT}}\left(1+k_{\text {бал }}\right)}{2 \cdot 10^{3}}
$$

где $k_{\text {Квд }}$ - учитывает включение в схему стандартного МОД компрессора высокого давления, нагнетающего газ в цилиндры ДВС при приближении поршней к верхней мёртвой точке $\left(k_{\text {Квд }}=1,02\right)$.

Среднечасовой расход ПГ соответствует базовому варианту.

Сравнительный анализ расчётных моделей

Рассмотренные варианты комплектации СЭУ можно разделить по принципу грузообработки на две группы. Первая - без УПСГ: базовый и вариант 3; вторая (с УПСГ) - варианты 1 и 2. При соответствии вариантов условию, устанавливаемому формулой 2, варианты первой группы имеют скорость в 20 узлов; а для вариантов второй группы существует возможность снижения скорости до 18,9 узла (плечо рейса 5000 миль), до 18,7 узла на 8000 миль и т.д.

На рисунке 1 приводится расход топлива по типам для расчётных вариантов, приведённых в предыдущем разделе (плечо рейса - 5000 миль). 


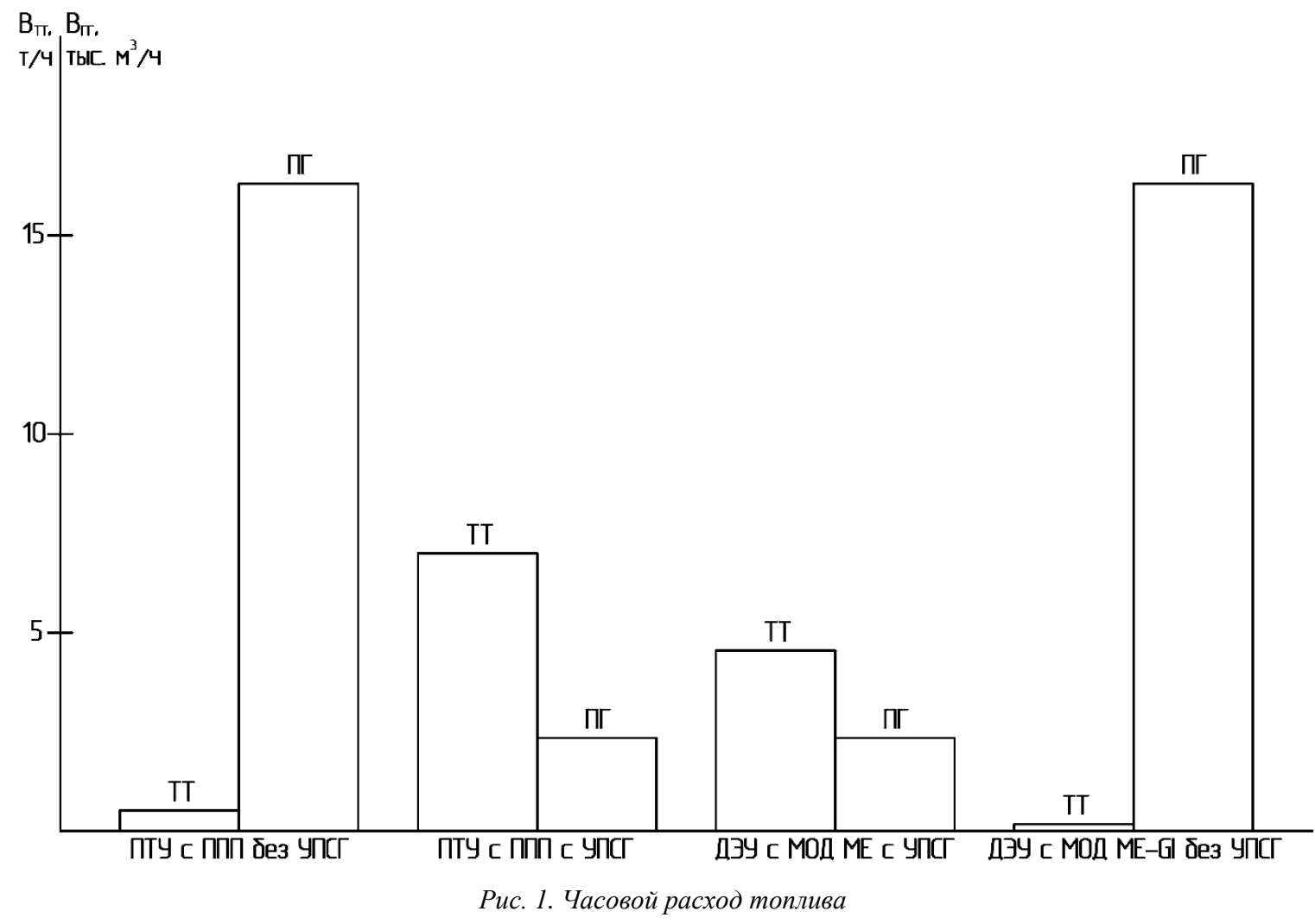

На рисунке 2 приведены графики зависимости принятого критерия эффективности от плеча рейса. Критерий определялся при средних ценах на типы топлива: $S_{\text {уд }}^{\text {TТ }}=324,90 \$ / \mathrm{HT}, \quad S_{\text {уд }}^{\text {ПГ }}=$ $316,54 \$$ тыс. нм$^{3}$.

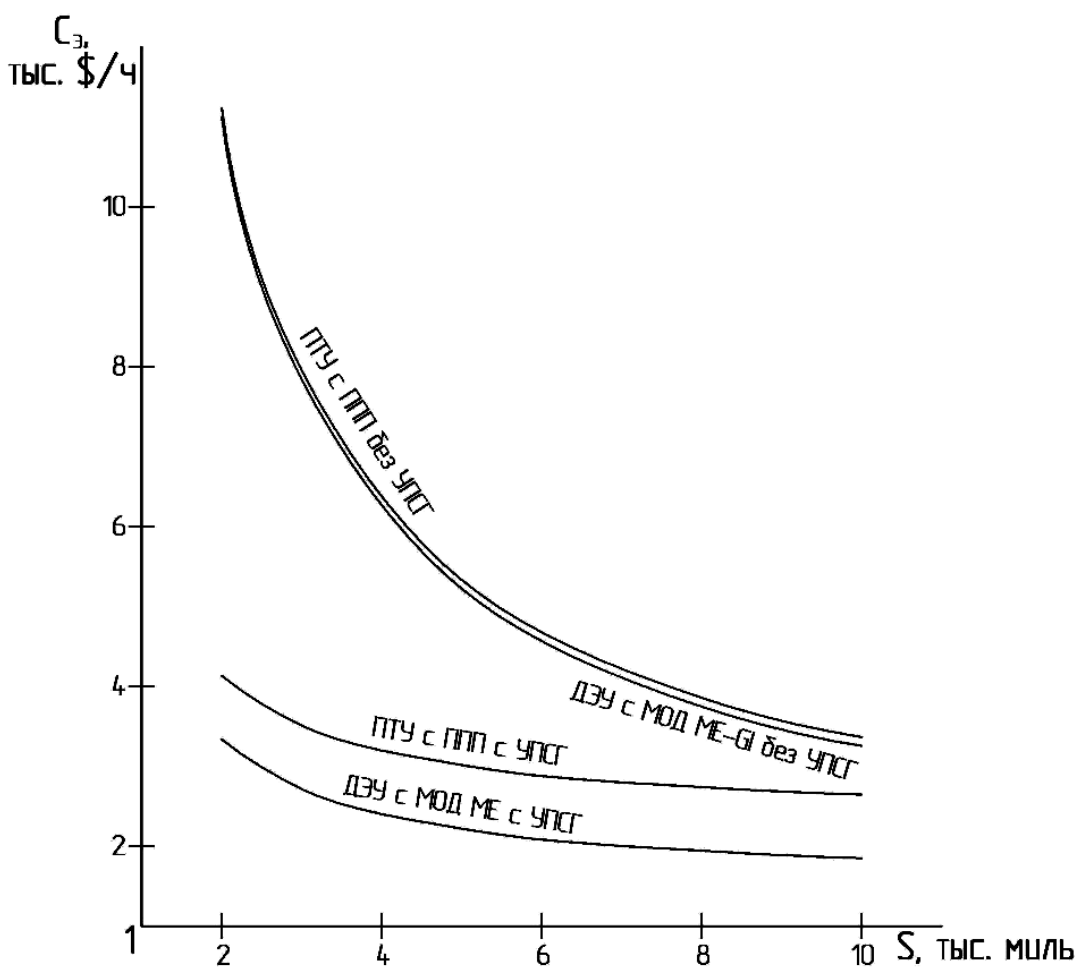

Рис. 2. Графики зависимостей $C_{\ni}=f(S)$

Из рисунка 2 следует, что рефрижераторные газовозы без УПСГ мало эффективны на коротком плече рейса вне зависимости от типа СЭУ. Это объясняется неэффективностью использования газа, оставляемого в танках на балластный переход. Значения критерия для вариантов первой группы 
различаются незначительно, ввиду того, что, несмотря на повышенную экономичность двигателя ME-GI, весь испаряющийся за рейс газ безвозвратно утрачивается для потребителя. На плече рейса в 5000 миль

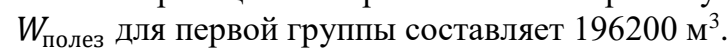

С другой стороны, как было отмечено ранее, бортовая УПСГ позволяет уменьшить объём газа, оставляемого на балластный переход, что уменьшает влияние этого фактора на эффективность судна в

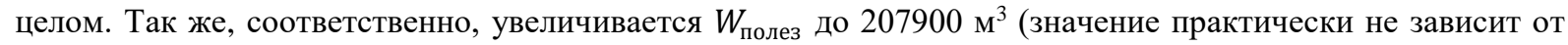
плеча рейса). Это, в свою очередь, обеспечивает снижение на $15 \%$ пропульсивной мощности и экономию топлива, даже несмотря на затраты энергии на УПСГ. При минимизации потерь ПГ и работе СЭУ на ТТ наблюдается существенное влияние типа ГД на эффективность газовоза.

\section{Сиисок литературы / References}

1. Romero Gomez J., Romero Gomez M., Ferreiro Garcia R., De Miguel Catoira A. On board LNG reliquefaction technology: a comparative study // Polish maritime research, 2014. № 81. C. 77-88.

2. Vorkapić A., Kralj P., Bernečić D. Ship systems for natural gas liquefaction // Multidisciplinary scientific journal of maritime research, 2016. № 30. C. 105-112.

3. Significant ships of 2007. London: Royal institution of naval architects, 2008. $126 \mathrm{c}$. 